Research paper

\title{
Investigation of stickiness influence in the anomalous transport and diffusion for a non-dissipative Fermi-Ulam model
}

\author{
André L.P. Livorati ${ }^{a, b, *}$, Matheus S. Palmero ${ }^{a}$, Gabriel Díaz-I ${ }^{a}$, Carl P. Dettmann ${ }^{b}$, \\ Iberê L. Caldas ${ }^{c}$, Edson D. Leonel ${ }^{\mathrm{a}}$ \\ a Departamento de Física, UNESP, Universidade Estadual Paulista, Av. 24A, 1515, Bela Vista, 13506-900, Rio Claro, SP, Brazil \\ ${ }^{\mathrm{b}}$ School of Mathematics, University of Bristol, Bristol, BS8 1TW, United Kingdom \\ ' Instituto de Física, IFUSP, Universidade de São Paulo, USP Rua do Matão, Tr.R 187, Cidade Universitária, 05314-970, São Paulo, SP, Brazil
}

\section{A R T I C L E I N F O}

\section{Article history:}

Received 30 January 2017

Revised 26 April 2017

Accepted 10 July 2017

Available online 18 July 2017

\section{Keywords:}

Stickiness influence

Anomalous transport and diffusion

Chaos

\begin{abstract}
A B S T R A C T
We study the dynamics of an ensemble of non interacting particles constrained by two infinitely heavy walls, where one of them is moving periodically in time, while the other is fixed. The system presents mixed dynamics, where the accessible region for the particle to diffuse chaotically is bordered by an invariant spanning curve. Statistical analysis for the root mean square velocity, considering high and low velocity ensembles, leads the dynamics to the same steady state plateau for long times. A transport investigation of the dynamics via escape basins reveals that depending of the initial velocity ensemble, the decay rates of the survival probability present different shapes and bumps, in a mix of exponential, power law and stretched exponential decays. After an analysis of step-size averages, we found that the stable manifolds play the role of a preferential path for faster escape, being responsible for the bumps and different shapes of the survival probability.
\end{abstract}

(c) 2017 Elsevier B.V. All rights reserved.

\section{Introduction}

Hamiltonian systems are typically non-integrable and non-ergodic [1-3], where their dynamics present mixed properties in the phase space, with KAM islands, invariant tori, spanning curves and chaotic seas. One of the main consequences of this mixed dynamics is the anomalous transport that a chaotic orbit may experience when passing near by stability regions. The orbit can stick to their boundaries, thus getting trapped around the bounded area of these islands and its cantori for a finite time (that could be long), in what it known as stickiness effect [4,5]. Applications of this trapping phenomenon can be found in many research areas as: fluid mechanics [6], plasma physics [7-9], celestial mechanics [10], acoustics [11], biology [12], among others (See Ref. [1,2] for reviews). This anomalous behavior serve as motivation for our study, where the interface between chaotic, quasi-periodic and stable dynamics is very complex and not yet fully understood and generates some open problems $[13,14]$.

\footnotetext{
* Corresponding author at: Departamento de Física, UNESP, Universidade Estadual Paulista, Av. 24A, 1515, Bela Vista, 13506-900, Rio Claro, SP, Brazil.

E-mail addresses: livorati@usp.br, andrelivorati@gmail.com (A.L.P. Livorati).
} 
A natural observable allowing the study of the statistical properties of the transport, in particular $\rho(n)$, the probability (given a suitable distribution of initial conditions) that an orbit does not escape through a hole until a time $n$. Here, the hole is defined as a predefined subset of the phase space. The most important aspect of this analysis is that the escape rate is very sensitive to the system dynamics. For strongly chaotic systems the decay is typically exponential [15-18], while systems that present mixed phase space the decay can be slower, presenting a mix of exponential with a power law [19-21], or even stretched exponential decay [22]. Indeed, when a non-exponential decay is observed the dynamics would require a long range correlation. This is a direct consequence of the stickiness influence in the dynamics.

The model under study in this paper is the Fermi-Ulam model (FUM). The FUM was proposed by Ulam in early60s [23] as an attempt to produce a prototype that could explain the Fermi Acceleration [24] (unbounded energy growth). The system consists of an ensemble of non interacting particles confined to move between two infinitely heavy walls, which the particles collide elastically. One wall is assumed to be fixed while the other one oscillates periodically in time. The phase space is mixed and contains periodic islands surrounded by a chaotic sea, which is limited by a set of invariant spanning curves $[15,25]$. This implies that we have a finite portion of the phase space for orbits to diffuse [15], which prevents the dynamics to exhibit unlimited diffusion in the velocity. The mechanics of the model leads to a complex variety of nonlinear phenomena in both conservative and dissipative dynamics [26-30]. From an experimental and quantum point of view, one can imagine the FUM as a schematic where an atom or a photon bounces under the influence of strong electromagnetic pulses, with applications in astrophysics [31], where the radiated energy represents a typical realization of an on-off intermittent process, atom-optics [32,33], quantum effects [34-36] and experimental devices [37,38], where atoms can be trapped in featured resonances by optical cavities and ultra cold potentials.

In this paper we investigate and seek to understand the stickiness influence in the transport for a non-dissipative FUM. Since the accessible phase space have a finite portion for the orbit to diffuse, it can be divided into two regions of high and low energy regimes, that depends on the initial velocity of the ensemble. In previous studies [15,25], only the lower ensemble of energy (basically composed by chaotic sea) was investigated, leaving aside the higher ensemble, which has more complicated dynamics with chains of islands, cantori and small portions of chaotic sea, and seems more interesting to be studied. So, in this paper we give focus to the higher ensemble, but not neglecting the lower one as well, yielding in a complete overview of the dynamical scenario for the FUM. Statistical analysis concerning the root mean square velocity shows that for both regimes we have a convergence to a steady state plateau for long time series. A transport analysis shows that there is stickiness in both ensembles, and it influences the decay rates of the survival probability, presenting different shapes and bumps in a mix of exponential, power law and stretched exponential decays. After an analysis of stepsize averages, we found that the stable manifolds play the role of a preferential path for faster escape, being responsible for the bumps and different shapes of the survival probability. These results give support to the stickiness influence towards to the anomalous transport and diffusion, where orbits can produce an extreme slower decay rates of the survival probability, with different bumps and shapes when compared with regular chaotic motion, and also can be extended to other similar dynamical systems.

The paper is organized as follows: In Section 2 we describe the details of the FUM mapping and some chaotic properties. Section 3 is devoted to the statistical analysis of the average velocity, as well as, the investigation of the anomalous transport and diffusion, concerning escape basins, survival probability curves and histogram of frequencies. Finally, in Section 4 we drawn some final remarks, conclusions and perspectives.

\section{The model, the mapping and chaotic properties}

In this section we will describe the model under study, so called Fermi-Ulam model (FUM), which consists of the motion of a free particle that suffers elastic collisions with two heavy walls, where one of them is said to be fixed at $x=l$, and the other one is periodic oscillating around $x=0$. Dissipation could be introduced in the system via inelastic collisions [39] where a damping coefficient can be considered on the walls [40]. Also, kinetic friction [41] and in flight dissipation [42] can be introduced as well. However, in this paper we will consider only the conservative version, where the collision with both walls are completely elastic. The dynamics of this system is described by a non-linear and measure preserving mapping for the variables velocity of the particle $v$ and time $t$ immediately after a $n$th collision of the particle with the moving wall.

There are two distinct versions of the dynamics description: the complete one, which consists in considering the complete movement of the time-dependent wall, and the simplified, that is often used to speed up numerical simulations, where the moving wall is set to be fixed, but the particle exchanges momentum and energy with it, as if the wall were normally moving. Both approaches produce a very similar dynamics considering conservative and dissipative cases [15,25]. We consider in this paper the complete version, whose the position of the vibrating wall is given by $x_{w}\left(t_{n}\right)=\varepsilon \cos w t_{n}$, where $\varepsilon$ and $w$ are respectively the amplitude and the frequency of oscillation.

The dynamics is described using a two-dimensional mapping, where the background formalism and mathematical tools backs to Pustyl'nikov [43]. We notice that are three control parameters, named $\ell, \varepsilon$ and $w$, and not all of them are relevant. We then define the following dimensionless and more convenient variables as: $V_{n}=v_{n} / w l, \epsilon=\varepsilon / l$ and measuring the time in terms of the number of oscillations of the moving wall $\phi_{n}=w t_{n}$. Starting with an initial condition $\left(V_{n}, \phi_{n}\right)$ with initial position of the particle given by $x_{p}\left(\phi_{n}\right)=\cos \left(\phi_{n}\right)$, the dynamics is evolved by a map $T$ which gives the pair $\left(V_{n+1}, \phi_{n+1}\right)$ in 
the $(n+1)$ th collision with the moving wall. We finally end up with the following mapping

$$
T:\left\{\begin{array}{l}
V_{n+1}=V_{n}^{*}-2 \epsilon \sin \left(\phi_{n+1}\right) \\
\phi_{n+1}=\left[\phi_{n}+\Delta T_{n}\right] \bmod (2 \pi)
\end{array}\right.
$$

The expressions for $V_{n}^{*}$ and $\Delta T_{n}$ depend on what kind of collision happens: (i) multiple collisions and; (ii) single collisions. The multiple collisions are such that, after the particle enters in the collision zone, $x \in[-\epsilon,+\epsilon]$ and hits the moving wall, before it leaves the collision zone, the particle suffers a second and multiple collision. It is also possible for the particle, depending on the combination of $V_{n}$ and $\phi_{n}$, suffers many other multiple collisions. In this case, the expressions for both $V_{n}^{*}$ and $\Delta T_{n}$ are given by $V_{n}^{*}=V_{n}$ and $\Delta T=\phi_{c}$.

The numerical value of $\phi_{c}$ is obtained as the smallest solution of an equation $G\left(\phi_{c}\right)=0$ with $\phi_{c} \in(0,2 \pi]$. Let us now discuss the origin of the function $G\left(\phi_{c}\right)$ and its physical implications. Between two collisions with the moving wall, the particle travels with a constant velocity since there is no gradient of potential between such collisions. Thus, the position of the particle is given by a linear equation in time. Besides, the motion of the moving wall turns out impossible to find an analytical expression of the instant of the impact. Therefore, the function $G\left(\phi_{c}\right)$ is obtained as an attempt to account the condition that the position of the particle is the same as the position of the moving wall at the instant of the impact. In this sense, the function $G\left(\phi_{c}\right)$ is written as

$$
G\left(\phi_{c}\right)=\epsilon \cos \left(\phi_{n}+\phi_{c}\right)-\epsilon \cos \left(\phi_{n}\right)-V_{n}^{*} \phi_{c} .
$$

If the function $G\left(\phi_{c}\right)$ does not have a root in the interval $\phi_{c} \in(0,2 \pi]$, we can conclude that the particle leaves the collision zone and a multiple collision no longer happens.

The same discussion used for the function $G\left(\phi_{c}\right)$ also holds when we consider the case of single collisions. The corresponding expressions used in the mapping in Eq. (1) are $V_{n}^{*}=V_{n}$ and $\Delta T_{n}=\phi_{r}+\phi_{l}+\phi_{c}$. The expression of $\phi_{r}$ denotes the time that the particle spends traveling to the right-hand side until it hits the fixed wall. The particle thus suffers an elastic collision and is reflected backwards with velocity $-V_{n}$, where the term $\phi_{l}$ denotes the time that the particle spends to enter the collision zone. These terms are given by $\phi_{r}=\left(1-\epsilon \cos \left(\phi_{n}\right)\right) / V_{n}$ and $\phi_{l}=(1-\epsilon) / V_{n}$. Finally, $\phi_{c}$ is numerically obtained as the smallest solution of the equation $F\left(\phi_{c}\right)=0$ with $F\left(\phi_{c}\right)$ given by

$$
F\left(\phi_{c}\right)=\epsilon \cos \left(\phi_{n}+\phi_{r}+\phi_{l}+\phi_{c}\right)-\epsilon+V_{n}^{*} \phi_{c} .
$$

Following the same line adopted for $G\left(\phi_{c}\right)$, Eq. (3) comes from the condition that the position of the particle is the same as that of the moving wall at the instant of the impact.

Both Eqs. (2) and (3) are exact expressions and come directly from the collision condition $x_{p}\left(t_{c}\right)=x_{w}\left(t_{c}\right)$, where $x_{p}$ and $x_{w}$ are respectively the equations of motion of the particle and the vibrating wall. The time $t_{c}$ is the collision time.

Fig. 1 shows the phase space for four different values of $\epsilon$, for 50 different initial conditions. One can see that the phase space presents basically the same mixed structure for all values of $\epsilon$. They have a chaotic sea in the low energy regime, and then a chain of islands appears as the velocity is increased. Also, the amount of stability islands in the phase space increases
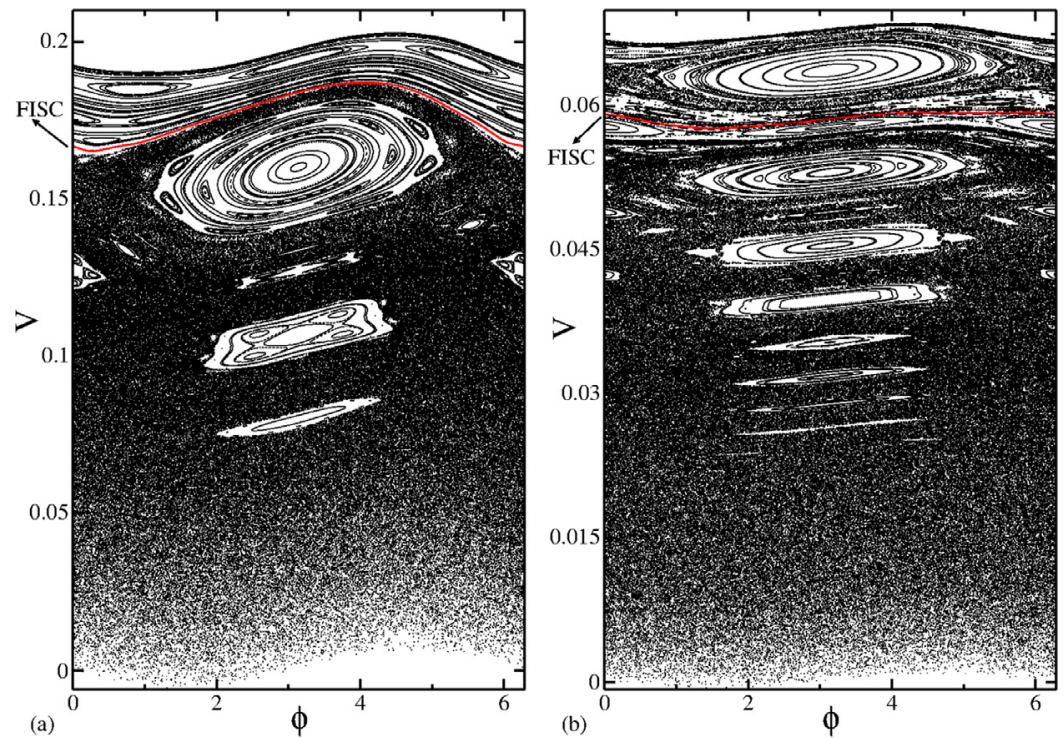

Fig. 1. Phase space for the complete dynamics of the FUM. In (a) $\epsilon=6 \times 10^{-3}$ and in (b) $\epsilon=8 \times 10^{-4}$. Also, we have depicted (in red) the position of the first invariant spanning curve (FISC). (For interpretation of the references to color in this figure legend, the reader is referred to the web version of this article.) 
as $\epsilon$ decreases. The limit of the growth of the chaotic sea is set by the first invariant spanning curve (FISC). The position of the FISC varies with $\epsilon$, and an analytical approach for its position can be found in Ref. [44]. Considering this approach for our model, we can set that the position of FISC is

$$
V_{\text {FISC }}=2 \sqrt{\frac{\epsilon}{K_{c}}} \approx 2 \sqrt{\epsilon},
$$

where $\epsilon$ is the control parameter and $K_{c}$ is the critical parameter value for the Chirikov Standard mapping [45], where the last invariant curve is destroyed, and there is a transition from local to global chaos. A similar transition can be also found in the bouncing ball model [19].

Observing Fig. 1, one can realize that depending of the initial condition, distinct kinds of dynamics may be observed. If one choose an initial velocity in the chaotic sea below the FISC, the particle will have access to the major region of the phase space, which is a limited region with an up barrier located in the region of the FISC, and a below limit set by $-\epsilon$. On the other hand, if one choose an initial velocity above the FISC, the dynamics can be stable, or even in a local chaotic region. The first scenario brings more complexity to the dynamics and is more interesting to investigate from the dynamical point of view, since we can find orbits that experience stickiness phenomenon as the time evolves, which may cause anomalous transport and diffusion in the dynamics.

One can ask about the fixed points and their stability. In fact, period-1 and period-2 fixed points were already characterized in previously studies $[15,46]$. For the period-1 fixed points, we have that the phase $\phi^{*}$, could be either equal to zero, for hyperbolic points, or equal to $\pi$, for the elliptical points, as one can check in Fig. 1 . For the velocity, we have that, $V^{*}=\frac{1-\epsilon \cos \left(\phi^{*}\right)}{m \pi}$, where considering the stability islands one finds $V^{*}=(1+\epsilon) / m \pi$. Also, this expression for the velocity fixed point, only holds if $m \geq \sqrt{\frac{1+\epsilon}{\epsilon \pi^{2}}}$ [15].

Also, regarding the determinant of the Jacobian matrix, one could ask if it should not be equal to unity, once the mapping dynamics is conservative. To answer this we must consider the extended phase space for the whole version of the model that considers four variables namely: (1) $x_{w}$ denoting the position of the moving wall; (2) $V_{p}$ corresponding to the velocity of the particle; (3) $E_{p}$ which is the energy of the particle and (4) the time $t$. The canonical pairs are: position and velocity $\left(x_{w}, V_{p}\right)$ and; energy and time $\left(E_{p}, t\right)$. As the way the mapping was constructed, the variables used are not canonical ones therefore the determinant of the Jacobian matrix in its conservative version should not be equal to the unit. However, the mapping preserves the following measure in the phase space $d \mu=[V+\epsilon \sin (\phi)] d V d \phi$.

\section{Results and discussion}

In this section, we made a statistical analysis for the dynamics of the FUM. Considering average properties of the quadratic velocity, we can "foreseen" the behavior of the velocity in an analytical way, and then compare it with the numerical results. We also calculate the decay of correlation times, also known as, the survival probability, for both opposite ensemble of energies, inside the limit region in the phase space. Stickiness shows itself inherent in the system, and affects the survival probability in a different manners, depending on the initial ensembles.

\subsection{Statistical analysis for the velocity}

Let us start by evaluating numerically the behavior of the root mean square velocity, which is made by considering $V_{R M S}=\sqrt{\overline{V^{2}}}$, where

$$
\overline{V^{2}}=\frac{1}{M} \sum_{i=1}^{M} \frac{1}{n} \sum_{j=1}^{n} V_{i, j}{ }^{2},
$$

where $M$ is the ensemble of initial conditions, and $n$ is the number of collisions (iterations). The average is taken along the orbit and along the ensemble of initial conditions. Also, the initial conditions where always chosen in the chaotic sea, uniformly distributed along the phase $\phi \in[0,2 \pi)$, considering two different regimes for the velocity: (i) lower energies,

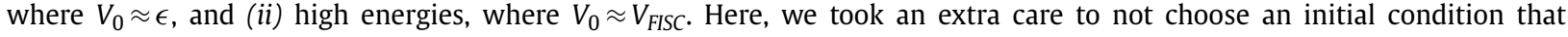
would lie inside a stability islands, otherwise it would damage the statistics.

In particular for (ii), the ensemble of initial conditions were chosen considering the following process. We started with a sample of low initial velocities, within a vector uniformly distributed in the phase $\phi \in[0,2 \pi)$ and evaluated the dynamics. After a collision, if the velocity was higher than in the previously one, we update the sample velocity. If not, the previously sample velocity remains. Such process was evaluated until $3 \times 10^{9}$ collisions. At the end, we have the highest possible velocity, within the accessible range of the phase space, and also very close to the position of the FISC.

Fig. 2 displays the $V_{R M S}$ curves evaluated over an ensemble of 2000 initial conditions, for high and low energy ensembles, iterated up to $10^{9}$ collisions with the vibrating platform. Bullets denote higher initial velocities $\left(V_{0}\right.$ around $\left.V_{\text {FISC }}\right)$ and the squares represent the lower energy ensemble $\left(V_{0}\right.$ around $\left.\epsilon\right)$. One can realize that for both ensembles of velocities, for the same control parameter $\epsilon$, the $V_{R M S}$ curves bend towards the same saturation plateau for $n \rightarrow \infty$, named $V_{S S}$ (steady state velocity). Also, it is significant to mention that the values of $V_{S S}$ are approximately half of the domain of the finite portion 


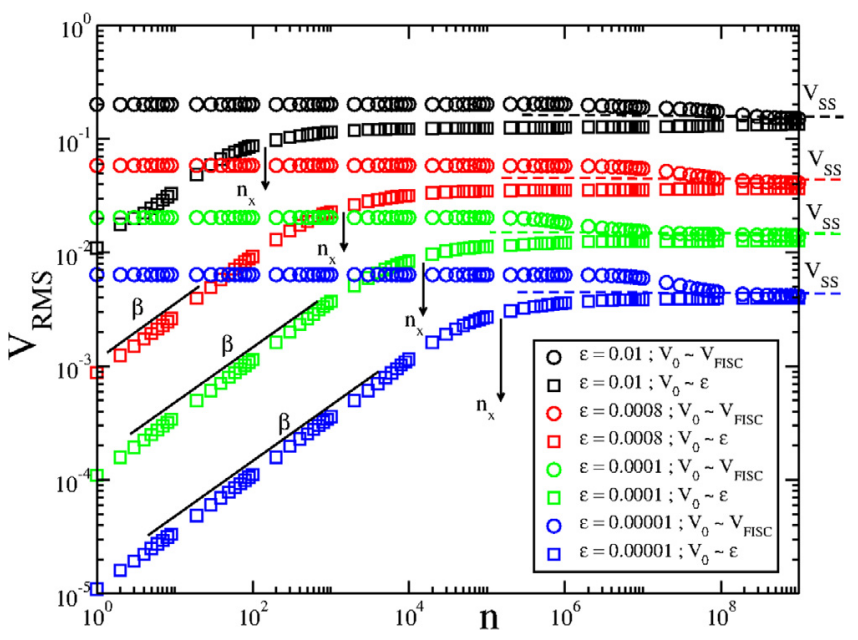

Fig. 2. $V_{R M S}$ curves of the FUM, for different values of $\epsilon$. For low energies, we have that the $V_{R M S}$ present a growing regime according to $\sqrt{n}$ for short times, then they suffer a crossover and evolve to a saturation plateau for long times. For high energies, $V_{R M S}$ curves have an almost constant behavior (slow linear decay) for short and medium times, and then they bend to a stationary state for long times. Also, the saturation plateaus for both regimes are the same, marked by the steady state velocity $V_{S S}$. (For interpretation of the references to color in the text, the reader is referred to the web version of this article.)

in the phase space, that a chaotic orbit can evolve. As said before, this finite portion is delimited by FISC and $-\epsilon$. Since, we can consider that FISC $\gg \epsilon$ we set that the stationary state velocity is

$$
V_{S S}=\frac{V_{F I S C}}{2}=\sqrt{\frac{\epsilon}{K_{c}}} \approx \sqrt{\epsilon},
$$

We can also write that $V_{S S} \propto \epsilon^{1 / 2}$, where the value $1 / 2$ coincides with the scaling exponent for the saturation plateau in previously studies of the FUM, concerning scaling formalism [25].

Let us now made an analytical analysis of the statistical properties of the velocity, taking the recurrence expression for the velocity of the mapping (1), taking the square of both sides and making an average, we have (see Ref. [15] for details)

$$
V_{R M S}=\sqrt{\left(V_{0}\right)^{2}+2 \epsilon^{2} n} .
$$

With the above expression we can "foreseen" the behavior of the $V_{R M S}$ curves, as shown in Fig. 2. Analyzing the $V_{R M S}$ curves one can see that basically those who have lower energy ensemble $\left(V_{0} \approx \epsilon\right)$, present a growth regime for short times, given by a power law of $n^{\beta}$, where $\beta \approx 1 / 2$; then they suddenly pass through a crossover $\left(n_{x}\right)$, and bend towards a saturation plateau $\left(V_{S S}\right)$ for long times. If we compare this low energy regime with the analytical expression of $V_{R M S}$ given by Eq. (7), we have that inside the square root, the term $2 \epsilon^{2} n>>\epsilon^{2}$, as the number of collision evolves. This explains why we have a growing regime in the velocity axis according to the power law of $V_{R M S} \propto\left(n \epsilon^{2}\right)^{\beta}$, confirming the analytical and numerical match of the results for short times.

Considering now the $V_{R M S}$ curves for the high energy ensemble $\left(V_{0} \approx V_{\text {FISC }}\right)$, one can observe a very smooth linear decay (almost constant) for initial times, and then it bend towards a decay for long times, followed by a stationary plateau $V_{S S}$. If we compare this behavior with the analytical expression of Eq. (7), where $V_{0} \approx V_{F I S C}$, we have that inside the square root, $4 \epsilon>>2 \epsilon^{2} n$ for short times. This explain the initial plateau almost constant, with very tiny linear decay. However, we will show latter in this paper that the duration of the almost constant initial plateau can be influenced by the stickiness, since orbits can be trapped for really long times in the high velocity regime.

Of course that the expression set in Eq. (7) is not valid for any $n$, particularly the larger ones. It is valid only for small $n$. Since the phase space is limited by invariant curves, an orbit cannot reach regions above the invariant curve for long time dynamics. If we literally take Eq. (7), as $n$ is increased, $V_{R M S}$ should also grows infinitely, and that is not what happens. So Eq. (7) has a range of $n$ where it is valid.

\subsection{Transport}

In this subsection we will address on the issues of the stickiness orbits influence in the dynamics of the FUM. We start evaluating the velocity distribution for both ensembles of energy, then a transport analysis is made, concerning the "time" until orbits reach the saturation plateau $V_{S S}$. Finally we study some properties of survival probability curves for both ensembles.

In order to understand better how stickiness orbits can influence the dynamics of the FUM, we initially construct a histogram of frequencies for the velocity, said $h(V)$ for both energy ensembles, as shows Fig. 3(a). We consider 10000 different 


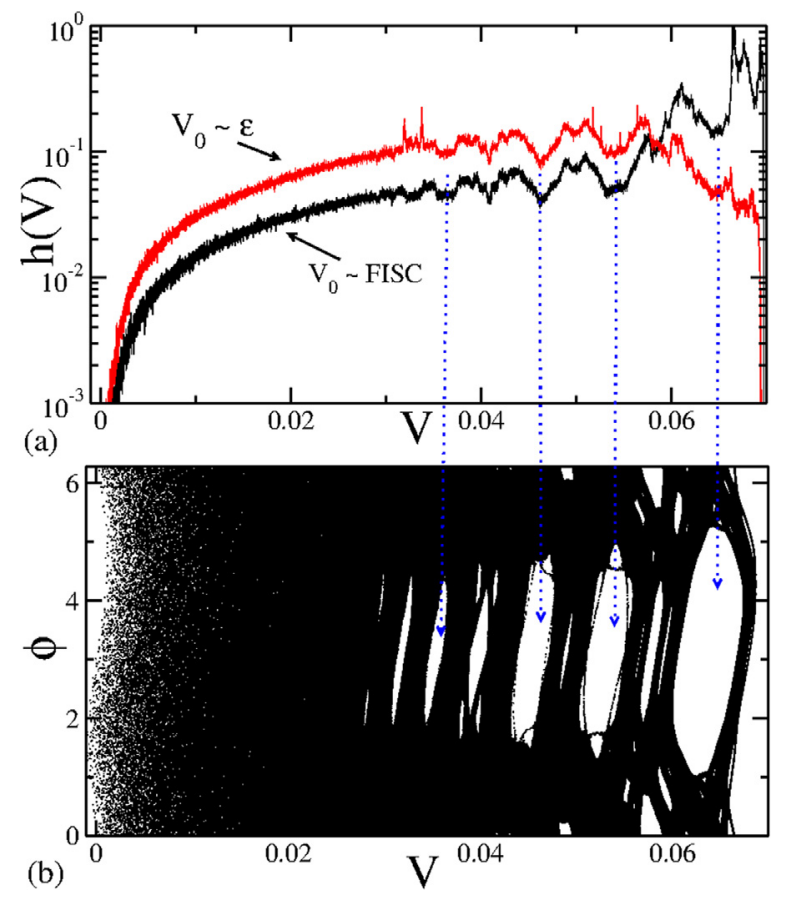

Fig. 3. Stickiness influence for $\epsilon=0.001$. In (a) we have the histogram of velocities $h(V)$ for the higher and lower ensembles. Although both ensembles access the whole phase space, lower initial velocities prefer to stay near $V_{S S}$, and higher initial velocities stay near the FISC, because they are trapped by stickiness. In (b) a comparison of the bigger stability islands with the "valleys" of $h(V)$.

initial conditions, evaluated up to $10^{6}$ iterations for a value of the control parameter $\epsilon=0.001$. Analyzing Fig. 3(a), one can see that for the lower ensemble, orbits access the whole phase space, but they do prefer staying in regions of medium velocity (near to $V_{S S}$ ). For the higher ensemble, orbits also access the whole phase space, but they prefer higher velocities to lower ones, particularly because of the stickiness influence. Also, a comparison between Fig.3(a and b), shows that there are some "valleys" in the histogram $h(V)$ for both ensembles. Such valleys perfectly match with the position of the bigger stability islands in the phase space, indicated by the arrows. With this in mind, for velocities very near the FISC values, we would expect several small valleys (as observed for $V_{0} \approx \epsilon$ ), since there is a huge chain of islands and cantori near the FISC. However this is not what happens. Instead of valleys, we do have peaks indicating that the orbits are most of the time trapped in stickiness. This also explains the long initial plateaus of the higher ensembles of the $V_{R M S}$ curves of Fig. 2 .

Since the investigation of the $V_{R M S}$ curves shown us that for long times both ensembles of initial velocity bend towards a saturation plateau around $V_{S S}$, we decided to investigate how the transport of both ensembles occur, by creating in somehow an escape basin. We created a grid of $1000 \times 1000$ initial conditions equally distributed in the whole accessible phase space, i.e., $\phi_{0} \in[0,2 \pi]$ and $V_{0} \in\left[-\epsilon, V_{F I S C}\right]$. Then each initial condition was evolved in time up to the limit of $10^{8}$ collisions with the moving wall or until the saturation velocity $V_{S S}$ were reached. In other words, we saved the iteration that the initial conditions took to reach the convergence plateau. This investigation of transport mechanism applies for the higher and the lower initial conditions ensemble.

Fig. 4 displays how this transport occurs for some values of the control parameter $\epsilon$. The color range denotes the number of collisions (plotted in logarithmic scale) that the particle had with the moving wall until reaching the escape velocity ( $V_{S S}$, indicated as a black line), and it can be interpreted as red (gray) indicating fast escape, to blue or black (black) denoting long time dynamics. For instance, a color scale marked as 10, represents exp(10), or about 22000 collisions until that initial condition reaches the saturation plateau in $V_{\text {hole }}=V_{S S}$. Also, white parts represent the stability islands and denote that the orbits did not reach $V_{S S}$ until $10^{8}$ collisions. One can also consider this dynamical evolution until the orbits reach the $V_{S S}$ plateau, as the introduction of a leakage, or a hole in the phase space [2,3], where orbits are allowed to "escape".

One can see in Fig. 4(a), for $\epsilon=0.01$, that initial conditions located near the $V_{S S}$ escape very rapidly in very fewer collisions for both ensembles. Looking specifically at the lower ensemble (below $V_{S S}$ ), we are able to see that in average, all initial conditions escape after around 200 collisions, in perfect agreement with the crossover collision $\left(n_{x}\right)$ displayed in Fig. 2 for the same $\epsilon$ control parameter. For the higher ensemble (above $V_{S S}$ ), we can depict two interesting behaviors. The first one concerns a preferential path in red (gray), among the stability islands, that is roughly similar to the shape of the manifolds for FUM. The other one is the trapping caused by stickiness effect around the stability islands, specially the ones near $V_{\text {FISC, }}$, where the color scale can reaches exp (18), or around $6.4 \times 10^{7}$, indicating that some of these orbits could never reach the saturation plateau for the time we evaluated the dynamics, or even taken an extremely long time to reach it. These results 


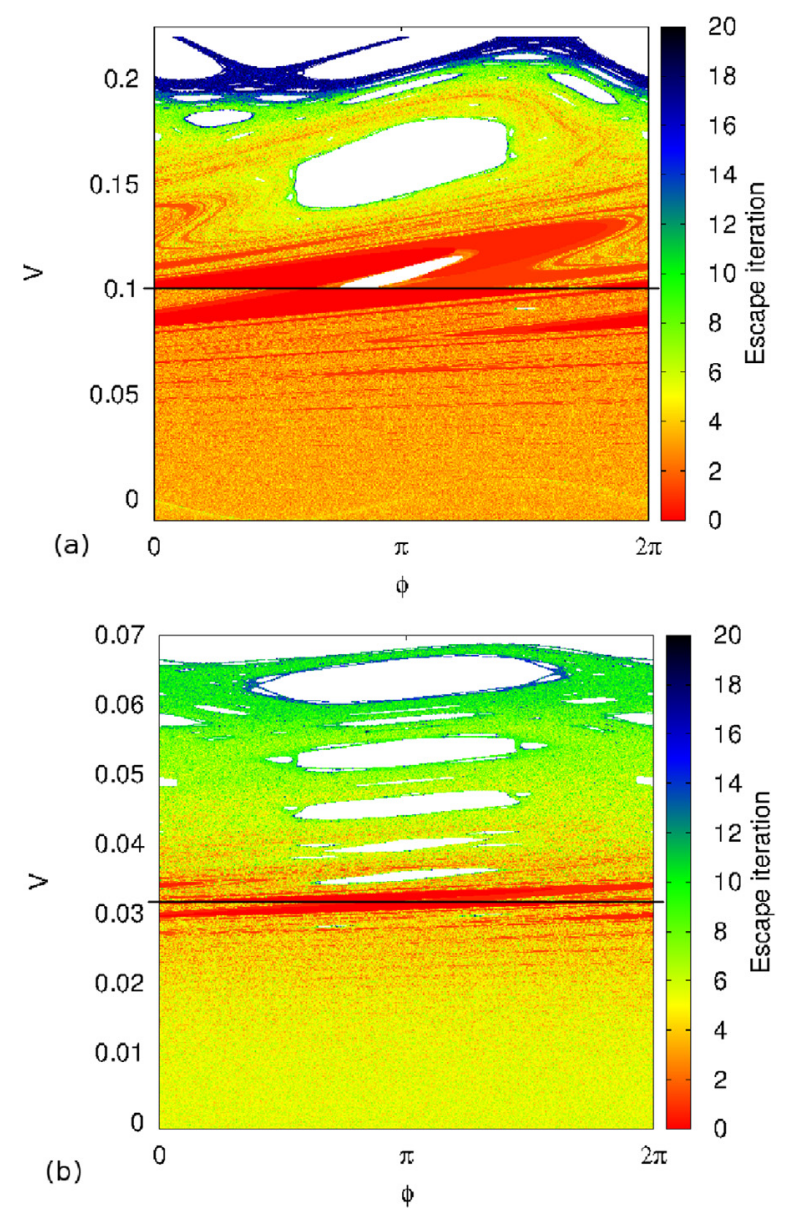

Fig. 4. Plot of the time evolution of initial conditions to reach the convergence plateau, or a hole in $V=V_{S S}$ (black line). In (a) we have $\epsilon=0.01$ and in (b) $\epsilon=0.001$. Dark blue (black) indicates long time evolution until reaching the hole, while red (gray) indicates fast escape. White denotes the particle never escaping until $10^{8}$ collisions. Particularly one can see that stickiness delays the escape influencing in the transport. (For interpretation of the references to color in this figure legend, the reader is referred to the web version of this article.)

also explain the long plateaus observed near the FISC in Fig.2, and furnish evidence of the stickiness influence in the dynamics. The same applies for Fig. 4(b), for $\epsilon=0.001$. The faster escape occurs for the initial conditions located near $V_{S S}$ for both ensembles, and in the lower ensemble, the average escape iteration is around exp(7), which is around 1100 collisions. Also, in the higher ensemble, we can see the stickiness influence, causing delay in the transport for initial conditions located near the stability islands and near the FISC.

\subsection{Survival probability}

Since we know that the dynamics will bend towards a convergence plateau $V_{S S}$ for long times, we introduce a distinct hole in the velocity for both ensembles of energy. For the lower velocity ensemble, we set a hole at $V_{\text {hole }}=V_{S S}=\sqrt{\epsilon}$, named $H_{d}$ (hole down). On the other hand, for the high energy ensemble, we place an escape region at $V_{\text {hole }}=1.2 V_{S S}$, called $H_{u}$ (hole up). Just for note, since orbits near FISC would take a really long time to reach $H_{u}$, in order to not "harm" the statistics, we place the $H_{u}, 20 \%$ above the $V_{S S}$.

Basically, the dynamics with the introduction of a hole follows: in the low energy ensemble, we consider that an initial condition had escaped, if its velocity is equal, or higher than $V=H_{d}$; in the same manner, for the higher energy ensemble, an initial condition escapes if its velocity is equal or lower than $V=H_{u}$. For both ensembles, we save in a vector the iteration in which the orbit had escaped, and then we build a frequency histogram for the escape, according the escape iteration.

The survival probability, described in terms of escape formalism [2,3], is then obtained by the integration of this escape frequency histogram, as

$$
\rho(v, n)=\frac{1}{N} \sum_{j=1}^{N} N_{r e c}(n),
$$


where the summation is taken along an ensemble of $N=5 \times 10^{6}$ initial conditions chosen along the chaotic sea for both ensembles of energy and $v$ is set as the escape velocity, or the hole position in the velocity axis. The term $N_{\text {rec }}(n)$, denotes the number of initial conditions that did not escape through the holes until the nth collision [3]. Also, the initial conditions were set uniformly distributed along the phase $\phi \in[0,2 \pi)$, the velocity is $V_{0}=1.1 \epsilon$ for the lower ensemble, and $V_{0}=V_{F I S C}$ for the higher ensemble, where we took an extra care to not chose any initial conditions that belong to a stability island, or otherwise the statistics would be damaged.

As it is known from the literature [3], the decay rate of $\rho(v, n)$ is extremely sensitive to the dynamics of the system. For strongly chaotic systems, which present normal diffusion, the decay is typically exponential [16-18], while systems that present mixed phase space, with irregular diffusion due stickiness influence, the decay can be slower, presenting a mix of exponential with a power law [19-21], or stretched exponential decay [22]. For the dynamics of the FUM, which has mixed properties in the phase space, the curves of $\rho(v, n)$ may present different behaviors, as mix of stretched exponential and power law for instance, depending of the value of $\epsilon$, the ensemble of initial conditions and the position of the hole.

Let us start investigating the escape rates for the lower ensemble. Fig. 5(a), shows the $\rho(v, n)$ curves when the escape in the velocity is considered through $H_{d}=V_{s s}$ for some values of $\epsilon$. Basically, one can observe that the decay rate is composed of two behaviors. For short and medium times, it obeys an exponential decay as

$$
\rho(v, n)=A \exp (-\zeta n)
$$

where $A$ is a non-negative constant and $\zeta$ is the decay rate. For long times, one can see it obeys a slower decay, that could be set as a power law according to

$$
\rho(v, n)=B n^{-\gamma},
$$

where $B$ is also a non-negative constant and $\gamma$ is the power law decay rate, that according to the literature [2,19,20], is found in a range of $\gamma \in[1,3]$. The slower decay can be also related to a stretched exponential decay, set up by

$$
\rho(v, n)=C \exp \left(-\mu n^{\alpha}\right)
$$

where $C$ is non-negative, $\eta$ is the decay rate, and $\alpha$ is the stretched exponent, that may be in the range of $\alpha \in[0,1]$, according Ref. [22].

For short and medium times, an exponential fit is in a good agreement to describe the behavior of $\rho(v, n)$, where we may obtain the decay rate $\zeta$, as shows Fig. 5(a). Also, in Fig.5(b), we display the behavior of $\zeta$ as a function of $\epsilon$. The best fit is a power law function, of a type $\zeta=C_{1} \epsilon^{z}$, where $C_{1}=2.9838(1)$ and $z=0.928(2)$. With the value of the $z$ exponent in hands, we may rearrange the horizontal axis as $n \rightarrow n \epsilon^{z}$, and obtain a perfect overlap of all $\rho(v, n)$ curves displayed in Fig. 5(a), indicating that the exponential decay rate is scaling invariant in the lower energy ensemble.

Another remarkable point, is that the end of the exponential decay rate curve for each $\epsilon$, matches with the crossover collisions $n_{x}$. For instance, one can take $\epsilon=0.001$, where according to Fig. 2 the crossover is about 2000 collisions. Comparing with Fig. 5(a), for the same $\epsilon$ (shown in red squares), the end of the exponential decay rate is also about 2000 collisions, which gives robustness to our statistical results.

One could ask now, about the slower decay rate. Indeed, even for a high number of initial conditions in the lower ensemble $\left(5 \times 10^{6}\right)$, only very few of them produce this slower escape (around 25 data points), as one can see in Fig. 5 (a), marked by the final tails of the $\rho(v, n)$ curves. So, the statistics becomes poor for us to affirm that the behavior of the tail is a power law or a stretched exponential. However, we can assume for sure, that they are slower than the exponential, indicating influence of some delay of theses orbits in escaping, which is due stickiness orbits. Also, one can see in Fig. 5(c) that this slower decay tails, are not scaling invariant, since that they might be produced by different chains and islands shapes as we range $\epsilon$.

Moving forward now, let us focus in the high ensemble of velocities. The escape velocity is setup as $V_{\text {hole }}=H_{u}=1.2 V_{S S}$, where this value was chosen considering the behavior of the $V_{R M S}$ curves in Fig. 2 . Since, the curves take really long times to reach $V_{S S}$ (about $10^{7}$ collisions, or even more), we decided to choose the hole position in the velocity axis a little bit above $V_{S S}$, so this would speed up numerical simulations and increase the statistics. The ensemble has $5 \times 10^{6}$ initial conditions, that were chosen very close to the value of the FISC, uniformly distributed along the phase $\phi \in[0,2 \pi)$.

Fig. 6(a) shows how the decay rate of $\rho(v, n)$ behaves for some values of $\epsilon$. One can see that, for almost all values of $\epsilon$ the decay rates show themselves in different ways, as "fast" initial decay, that seem roughly to follow exponential decay, and for medium and long times, we observe "bumps" of slower decays. One can also see that, for some values of $\epsilon$, there are still an amount of initial conditions, that did not escaped yet, until $10^{7}$ collisions, indicating a strong delay in the transport along the dynamical evolution.

In order to reveal the nature of the decay rates of the $\rho(v, n)$ curves, we made use of a nonlinear curve fitting in some curves of Fig.6(a), according Eqs. (9)-(11). According the literature [22], when one has a stretched exponent decay, the exponent $\alpha$ should be in a range of $\alpha \in[0,1]$, and also, if we observe this kind of decay, slower then the regular exponential one, it is evidence of stickiness phenomenon influence in the dynamics. Considering that, one can look at Fig. 6(b and c) and realize that the decay rates for $\rho(v, n)$ follow in a best fit evaluation, two consecutive stretched exponential decays for short and medium times, and a power law for really long times, for respective $\epsilon=0.08$ and $\epsilon=0.06$. Their respective $\alpha$ and $\gamma$ values are drawn in caption of the figure. Also, the value of $\gamma$ is inside the range considered to be acceptable in the literature $[2,19,20]$, and also indicates stickiness influence in the dynamics. However, for other values of $\epsilon$, one can 

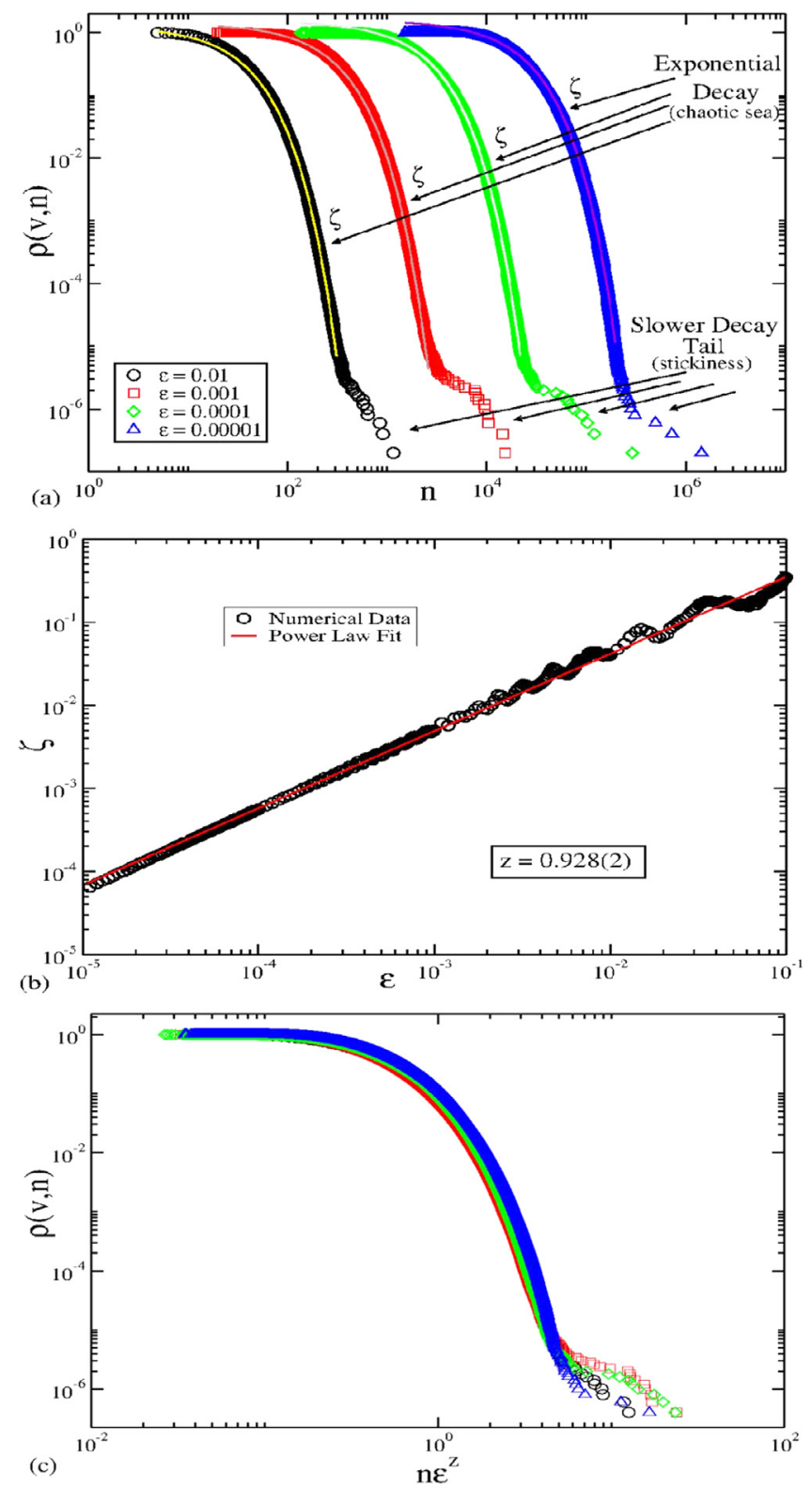

Fig. 5. (a) Plot of the survival probability curves $\rho(v, n)$ for some values of $\epsilon$, where the escape velocity is set as $H_{d}=V_{S S}$, for the lower ensemble of energy. The curves experience an exponential decay, according $\rho(v, n) \propto e^{-\zeta n}$. By their final tail, there is a slower decay, that is due stickiness orbits. In (b), we have the $\zeta$ exponent plotted as function of $\epsilon$. A power law fitting furnishes us a good agreement with an exponent $z=0.928(2)$. And in (c) we display a scaling invariance of the survival probability curves and its perfect overlap regarding the $z$ exponent obtained in (b).

find different combinations of decay rates of $\rho(v, n)$. In Fig. 6(d), for $\epsilon=0.004$, we can evaluate three successive different stretched exponential fits, while in Fig.6(e), we have a regular exponential for short times, then a stretched exponential decay, followed by a power low decay for medium and long times.

Such peculiar behavior of the decay rate of $\rho(v, n)$ curves displayed in Fig. 6, such as successive stretched exponential decay rates, and combinations of stretched exponential and power laws, indicates how can sticky orbits delay the transport for the high energy ensemble. Such slower decays, were indeed expected, since the initial conditions were chosen in a region of high concentration of stability islands, where the particle "prefers to stay" during the dynamics, as also shown in Figs. 3 and 4. Also, one could ask about the nature of the exponent $\alpha$ and its relation with the parameter $\epsilon$. In fact, we cannot assume for sure its dependence. Our supposition, is that such dependence has to do with the fractality of the system, and maybe the configuration and amount of stability islands in the accessible region for the particle. Yet, it still remain as an open issue. 

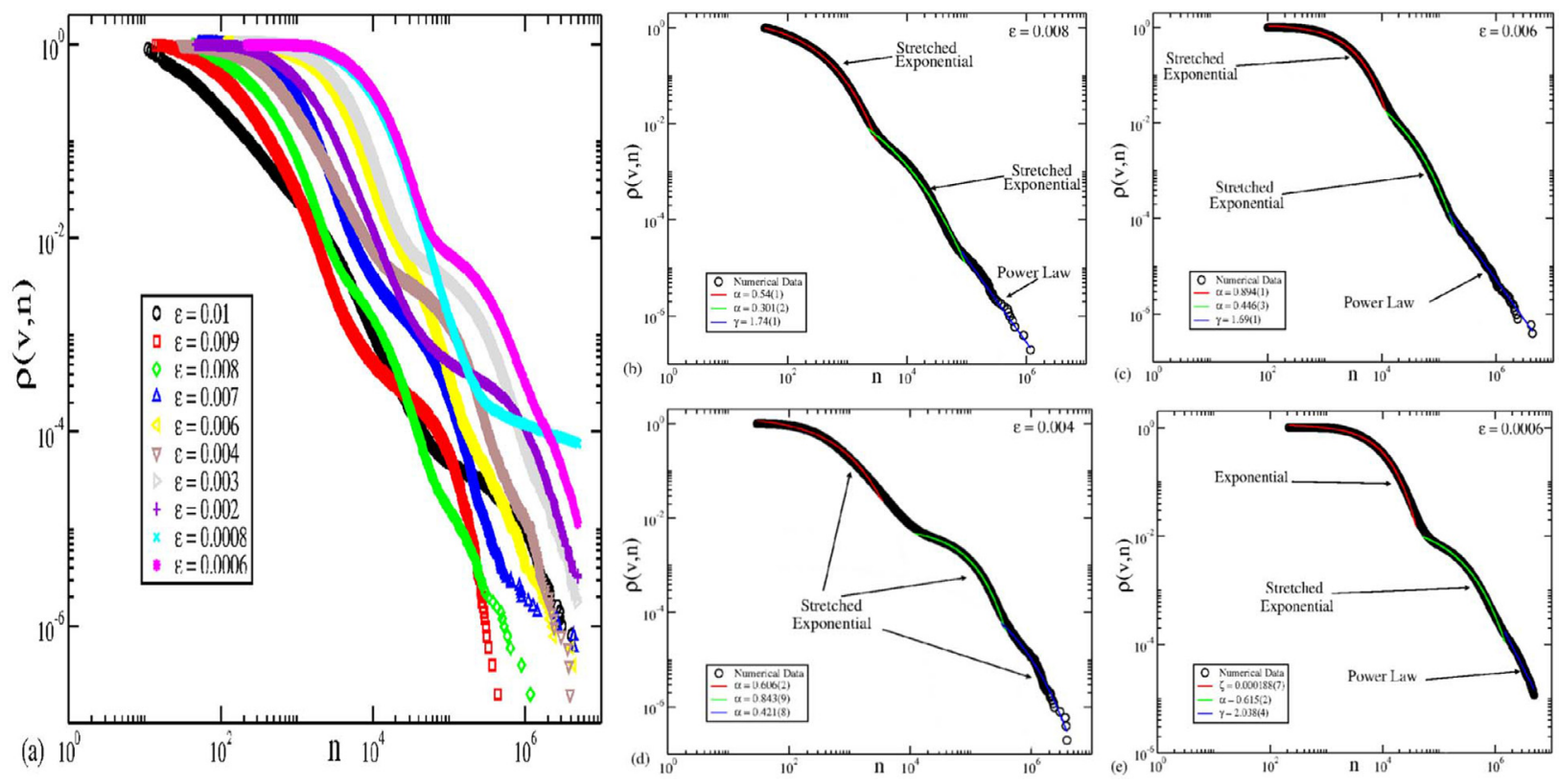

Fig. 6. (a) Plot of the decay rate $\rho(v, n)$ for some values of $\epsilon$, considering the hole in the velocity axis as $H_{u}=1.2 V_{S S}$. In (b) and (c), a nonlinear curve fitting furnishes two successive stretched exponential fits, and a final power law tail; in (d) we have three successive stretched exponential decays; and finally in (e) we have an exponential decay, followed by a stretched exponential and a power law tail. The values for the decay exponents $\alpha, \gamma$ and $\zeta$, are labeled in their respective items.

Also, as an attempt to complement the study of the transport for the high energy ensemble, we selected some very special initial conditions, in the decay rate of $\rho(v, n)$. We selected those who produce a fast escape rate, i.e., those located at the beginning of the decay of $\rho(v, n)$; and those who are located in the "bump" of the stretched exponential decay. With those initial conditions in hand, we evaluated a step-average over the velocity, in order to locate possible trappings in some particular regions of the phase space.

Fig. 7 shows the behavior of $\langle V\rangle_{100}$ vs. $n$ for some initial conditions from the high ensemble energy until they reach the escape velocity set as $V_{\text {hole }}=0.14$ for $\epsilon=0.008$, considering an average of step-size 100 in their orbits. From Fig. 7(a-d) we display the initial conditions chosen in the beginning of the escape rate. One can see that the orbits spent very short time in the dynamical evolution (about 4000 or less collisions) before escaping. Also, we marked some stickiness regime observed during the dynamics. Concerning now, the long range orbits, Fig. $7(\mathrm{e}-\mathrm{h})$ show their dynamical evolution until they reach the hole. These orbits take extremely long times to reach the hole (about $4 \times 10^{5}$ collisions), experiencing lots of stickiness influence during their dynamics.

Another remarkable point concerning Fig. 7, is that both, fast and long range orbits, seem prefer to stay in the upper layer (near the FISC) confined between $V \approx 0.18$, before escaping. Also, after leaving this region in the phase space, the orbits escape quickly. We believe that the reason for such rapid escape occur lies in the stable manifold, that seems to drawn a preferential path for the orbits to escape. Such phenomena was also observed in the bouncing ball model [47].

Fig. 8 displays a zoom-in view of the transport of orbits until they escape considering the high energy ensemble for $\epsilon=0.008$. The escape basin, was set up considering a grid of $1000 \times 1000$ initial conditions uniformly distributed along the phase $\phi \in[0,2 \pi)$ and velocity $V \in\left[0.17, V_{F I S C}\right]$. The escape velocity was set as $V_{\text {hole }}=0.14$, and the color gradient indicates the iteration of escape from red and orange (light gray) indicating fast escape, to blue and black (black) indicating long range iterations. White regions denotes that the orbit never escaped.

Together with the escape basin, we plotted as black lines, a piece of the stable manifold for the highest saddle point in the accessible phase space located at $m=2$. One can see that the drawn of the stable manifold (black lines) remarkable coincides with the regions of fast escape painted in red and orange (light gray), which is a clear evidence, that the stable manifold is responsible for the preferential path for the escape of orbits, and also explains the rapid escape after a long trapping regime of the orbits displayed in Fig. 7. Yet, in Fig. 8 we have also a clear influence of stickiness orbits colored in blue and black (black), near the major island and its cantori, and in the first layer, very close to the FISC. Basically, an orbit evolving into the strong stickiness regime layer (near FISC), may leave in somehow the region delimited by these layers, and reach one of the manifold branches, thus leading the orbit to escape. So, the stable manifold seems to play a crucial role, in which orbit would escape faster or slower.

Our best guess is that maybe the number of crossings between the manifolds (like a homoclinic tangle), would somehow influence the transport, producing rapid and slow escape rates depending on the number of crossings. However, this supposition still needs further investigation. 

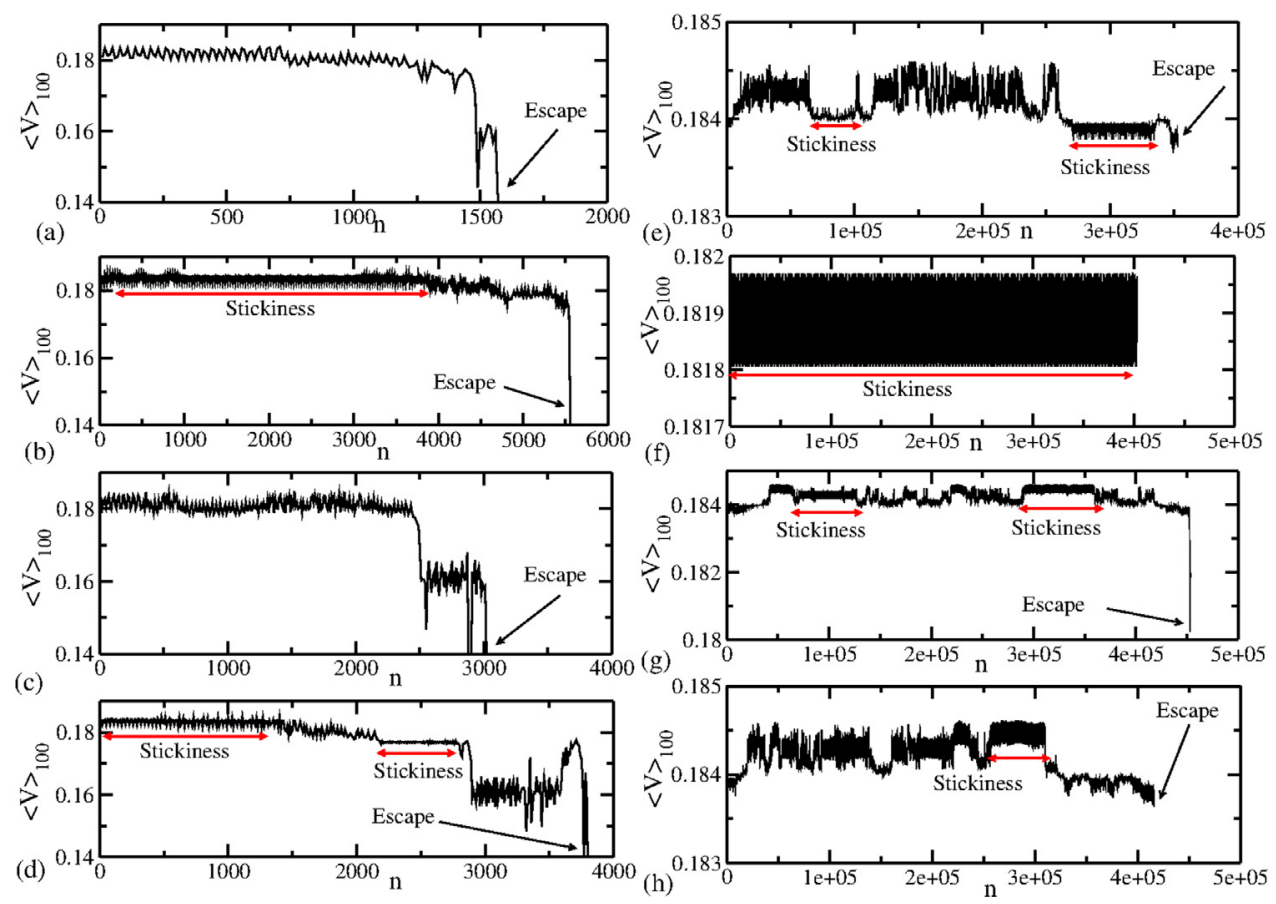

Fig. 7. Behavior of $\langle V\rangle_{100}$ vs. $n$ for different values of initial conditions for $\epsilon=0.008$. In (a), (b), (c), and (d), we have fast escape orbits, and in (e), (f), (g) and (h), we have delayed escape orbits. Also, some stickiness regime during the time evolution are drawn for fast and delayed escape orbits.

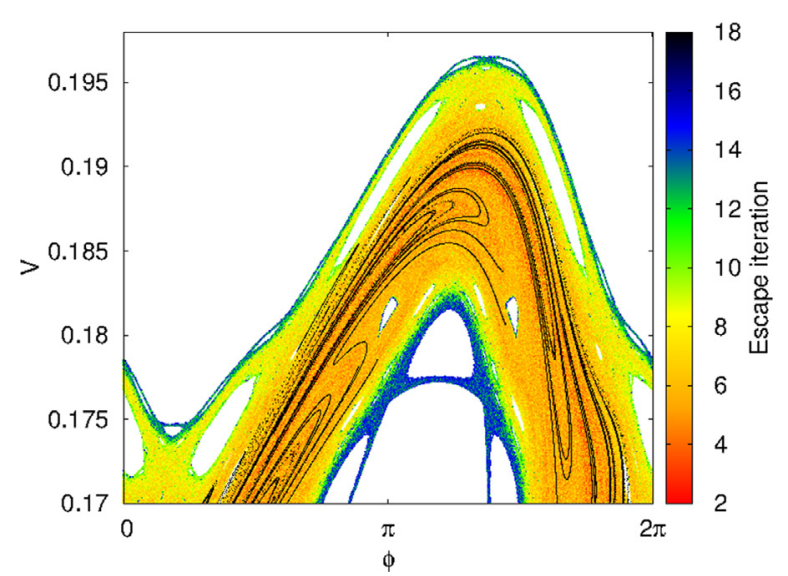

Fig. 8. Plot of $V$ vs. $\phi$ for the escape basin concerning $\epsilon=0.008$, where red and orange (light gray) indicate fast escape and blue and black (black) denote long times until escaping. The stable manifold for the saddle $m=2$ (black lines) draws a preferential path for the escaping orbits. (For interpretation of the references to color in this figure legend, the reader is referred to the web version of this article.)

\section{Final remarks and conclusions}

To summarize, we have investigated the dynamics of a particle undergoing elastic collisions in a domain composed by two heavy walls, where one is fixed and the other is periodically moving in time. A nonlinear, measure preserving mapping was obtained and a mixed phase space was characterized composed by a chaotic sea, KAM islands and an upper first invariant spanning curve (FISC), that works as a barrier, preventing the chaotic sea to grow.

Statistical analysis for different ensembles of initial conditions (high and low velocity) leads the root mean square velocity to the same steady state for long times through different paths, which may be directed influenced by stickiness. A transport investigation shows us that orbits in the lower ensemble reach the saturation plateau faster due to lack of stability islands in the lower portion of the chaotic sea, while the higher ensemble prefers to stay near the region of the FISC, where it is affected by the influence of stickiness and anomalous diffusion due to the huge amount of stability islands in that region, which causes a delay in the transport where the orbits could stay trapped for extremely long times. 
Evaluating an investigation of the survival probability, the lower ensemble presents typical exponential decay rates and they are scaling invariant with respect to the control parameter. For the higher ensemble, the decay rates show themselves with very unusual decay rates with bumps and different shapes, as successive stretched exponential decays, followed by power law tails, which mark a direct influence of sticky orbits in the dynamics. Evaluating a step-size average over orbits that face long time correlations, we find out that the stable manifolds play a crucial role in the dynamics, outlining an escape path for rapid escape in the higher ensemble, influencing which orbit would escape faster or slower.

In the near future it would be interesting to investigate the crucial role of the manifolds very near the FISC for different saddles of different periods, focusing in the number of crossings between them. As well, an analysis of the stretched exponential exponent, and its relation with the preferential chains of islands that orbits may prefer to stay trapped seems promising. One might also try an approach via thermodynamics formalism to describe the convergence to the steady state plateaus for both ensembles.

\section{Acknowledgments}

ALPL acknowledges FAPESP (2014/25316-3) and FAPESP (2015/26699-6) for financial support. MSP thanks to FAPESP (2014/27260-5), GDI thanks Brazilian agency CAPES, CPD thanks to EPSRC (EP/N002458/1), ILC thanks FAPESP (2011/192961) and CNPq, EDL thanks FAPESP (2012/23688-5) and CNPq (303707/2015-1). ALPL also thanks the University of Bristol for the kindly hospitality during his stay in UK. This research was supported by resources supplied by the Center for Scientific Computing (NCC/GridUNESP) of the São Paulo State University (UNESP).

\section{References}

[1] Meiss JD. Chaos 2015;25:097602.

[2] Altmann EG, Portela JSE, Tél T. Rev Mod Phys 2013:85:869.

[3] Gaspard P. Chaos, scattering and statistical mechanics. Cambridge: Cambridge University Press; 1998.

[4] Zaslasvsky GM. Physics of chaos in Hamiltonian systens. New York: Imperial College Press; 2007.

[5] Zaslasvsky GM. Hamiltonian chaos and fractional dynamics. New York: Oxford University Press; 2008.

[6] Solomon TH, Weeks ER, Swinney HL. Phys Rev Lett 1993;71:3975.

[7] del Castillo-Negrete D, Carreras BA, Lynch VE. Phys Rev Lett 2005;94:065003.

[8] Kroetz T, Roberto M, Silva ECd, Caldas IL, Viana RL. Phys of Plasmas 2008;15:092310.

[9] Portela JSE, Caldas IL, Viana RL. Eur Phys J Spec Top 2008;165:195.

[10] Contopoulos G, Harsoula M. Celest Mech Dyn Astr 2010;107:77.

[11] Altmann EG. Phys Rev A 2009;79:013830.

[12] Tél T, de Moura A, Grebogi C, Károlyi G. Phys Rep 2005;413:91.

[13] Bunimovich LA. Nonlinearity 2008;21:T13.

[14] Dettmann CP. "Recent Advances in Open Billiards with Some Open Problems", Frontiers in the study of chaotic dynamical systems with open problems. World Scientific; 2011.

[15] Livorati ALP, Dettmann CP, Caldas IL, Leonel ED. Chaos 2015;25:103107.

[16] Leonel ED, Dettmann CP. Phys Lett A 2012;376:1669.

[17] Méndez-Bermúdez JA, Martínez-Mendoza AJ, Livorati ALP, Leonel ED. J Phys A 2015;48:405101.

[18] Livorati ALP, Georgiou O, Dettmann CP, Leonel ED. Phys Rev E 2014;89:052913.

[19] Livorati ALP, Kroetz T, Dettmann CP, Caldas IL, Leonel ED. Phys Rev E 2012;86:036203.

[20] Altmann EG, Tél T. Phys Rev E 2009;79:016204.

[21] de Oliveira JA, Dettmann CP, Costa DRd, Leonel ED. Phys Rev E 2013;87:062904.

[22] Dettmann CP, Leonel ED. Physica D 2012;241:403.

[23] Ulam SM. A collection of mathematical problems. Interscience tracts in pure and applied mathematics. New York-London: Interscience Publishers; 1960. no. 8.

[24] Fermi E. Phys Rev 1949;75:1169.

[25] Leonel ED, McClintock PVE, da Silva JKL. Phys Rev Lett 2004;93:014101.

[26] Karlis AK, Papachristou PK, Diakonos FK, Constantoudis V, Schmelcher P. Phys Rev Lett 2006;97:194102.

[27] Karlis AK, Papachristou PK, Diakonos FK, Constantoudis V, Schmelcher P. Phys Rev E 2007;76:016214

[28] Oliveira DFM, Leonel ED. New J Phys 2011;13:123012.

[29] Oliveira DFM, Leonel ED. Physica A 2013;392:1762.

[30] Tavares DF, Leonel ED, Filho RNC. Physica A 2012;391:5366.

[31] Veltri A, Carbone V. Phys Rev Lett 2004;92:143901.

[32] Saif F, Bialynicki-Birula I, Fortunato M, Schleich WP. Phys Rev A 1998;58:4779.

[33] Steane A, Szriftgiser P, Desbiolles P, Dalibard J. Phys Rev Lett 1995;74:4972.

[34] Dembinski ST, Makowski AJ, Peplowski P. Phys Rev Lett 1993;70:1093.

[35] José JV, Cordery R. Phys Rev Lett 1986;56:290.

[36] Saif F, Rehman I. Phys Rev A 2007;75:043610.

[37] Kowalik ZJ, Franaszek M, Pieranski P. Phys Rev A 1988;37:4016.

[38] Warr S, Cooke W, Ball RC, Huntley JM. Physica A 1996;231:551.

[39] Diaz-I G, Livorati ALP, Leonel ED. Phys Lett A 2016;380:1830.

[40] Livorati ALP, Caldas IL, Dettmann CP, Leonel ED. Phys Lett A 2015;379:2830.

[41] Ladeira DG, Leonel ED. Phys Rev E 2010;81:036216.

[42] de Oliveira DFM, Leonel ED. Chaos 2012;22:026123.

[43] Pustyl'nikov LD. Russian Math Surv 1995;50:145.

[44] Leonel ED, de Oliveira JA, Saif F. J Phys A 2011;44:302001.

[45] Chirikov BV. Phys Rep 1979;52:263.

[46] Lichtenberg AJ, Lieberman MA. Regular and chaotic dynamics. Applied mathematical science, vol. 38. New York: Springer Verlag; 1992.

[47] Kroetz T, Livorati ALP, Leonel ED, Caldas IL. Phys, Rev E 2015;92:012905. 